\title{
Paternal Childcare In Early Childhood and Problematic Behavior in Children: A Population-Based Prospective Study in Japan
}

Manami Ochi ( $\sim$ ochi.m.aa@niph.go.jp)

National Institute of Public Health

Takeo Fujiwara

Tokyo Medical and Dental University

\section{Research Article}

Keywords: father, behavioral problem, parenting, early childhood, longitudinal data

Posted Date: April 7th, 2021

DOI: https://doi.org/10.21203/rs.3.rs-382771/v1

License: () (7) This work is licensed under a Creative Commons Attribution 4.0 International License. Read Full License 


\section{Abstract}

Background: Little is known about the effects of paternal childcare on children's behavioral development. This study explored the association between paternal childcare during infancy in terms of childcare hours and type of caregiving behavior and subsequent behavioral problems in children aged 5.5 years stratified by sex.

Methods: We analyzed data from the Longitudinal Survey of Newborns in the $21^{\text {st }}$ Century (2001-2006), a population-based cohort survey in Japan ( $\mathrm{N}=$ 27,870). Paternal childcare was assessed at 18 months in terms of paternal childcare hours on weekdays or weekends and the frequency of each type of childcare (feeding, changing diapers, bathing, putting the child to sleep, playing with the child at home, and taking the child outside). Six categories of child behavioral problems were assessed when the children were 5.5 years old. Logistic regression analysis was applied to account for the known confounding variables.

Results: Longer paternal childcare hours, on both weekdays and weekends in infancy, had a protective effect on behavioral problems at 5.5 years of age. The dose-effect relationships were found between the frequency of fathers taking their children outside and behavioral problems in boys, and the frequency of fathers playing with their children at home and behavioral problems in both boys and girls.

Conclusions: Paternal childcare during infancy could prevent subsequent behavioral problems in children. Several specific paternal caregiving behaviors, such as taking their children outside and playing with them at home, may play an important role in preventing subsequent behavioral problems.

\section{Background}

An increasing body of research has shown that paternal childcare in early childhood can contribute to child socioemotional development and well-being in a variety of ways [1]. While most studies focus on the importance of mother-infant interaction in childcare, increased involvement in childcare by fathers is associated with multiple aspects of child health and well-being, such as higher cognitive skills [2, 3], receptive language skills [3, 4], better anthropometric outcomes [5], improved social competence [6], higher educational attainment [7, 8], fewer injuries [9], and fewer behavioral problems [10-13].

Direct engagement with children includes caregiving behaviors, such as feeding, changing diapers, playing, and social activities that promote the sociopsychological development of children [1]. Childcare provided by fathers may lead to increased maternal relaxation time and improved maternal mental health and may eventually affect their interactions with their children. Therefore, future research needs to consider not only the duration but also the extent of paternal childcare [14].

Certain types of paternal involvement with children may be partly determined by the child's characteristics. For example, previous studies using path analysis found bidirectional associations between paternal involvement and child behavior, and a tendency for children with conduct problems or hyperactivity to have less involved fathers at subsequent ages $[15,16]$. Children's characteristics are also strongly associated with their developmental behaviors. Paternal childcare is responsive to the child's temperament or disabilities, and children with difficulties may elicit less paternal involvement [16, 17]; however, few studies have taken this into account.

Further, previous studies on paternal childcare and its effect on child behavior have been limited to Western countries [18]. Parental behavior is highly contextdependent and diverse depending on various societal factors: geographical conditions, family characteristics, economic status, work-related factors, societal norms and beliefs, and so on $[19,20]$. In Japan, where working and commuting hours are longer than in Western countries, many fathers are unable to spend a satisfactory amount of time with their children [21], and the impact of paternal engagement in childcare on children remains unclear.

This study aims to clarify the impact of paternal childcare during infancy on behavioral problems in later childhood in Japan, in terms of 1) childcare hours and 2) the type of caregiving in the Japanese population.

\section{Methods}

\section{Study sample}

We used data from the Longitudinal Survey of Newborns in the 21 st Century, a population-based survey conducted by the Ministry of Health, Labor, and Welfare in Japan. The study sample included all babies born in Japan between January 10 and 17 or July 10 and 17, 2001, using birth records from national vital statistics. The baseline survey was mailed to parents when their infants were six months old $(n=53,575)$. A total of 47,015 caregivers responded to the baseline questionnaire (response rate: $87.7 \%$ ). After the baseline survey, annual surveys were conducted by sending questionnaires to participants by mail. We used data from 2001,2002 , and 2006 survey waves. A total of 43,925 ( $93.4 \%$ ) caregivers responded in the 2002 survey wave, and 38,540 ( $82.0 \%$ ) responded in the 2006 survey wave. We included children who lived with both parents and children whose mothers answered the questionnaire to maintain consistency in the assessment of childcare (excluded samples $n=3,458,9.0 \%$ ). We excluded responses with missing data for the variables used in the analysis: child problem behavior $(n=1,443,3.7 \%)$, paternal involvement in childcare and domestic chores $(n=2,533,6.6 \%)$, maternal involvement in childcare and domestic chores $(n=1,277,3.3 \%)$, parental education $(n=578,1.5 \%)$, parental employment $(n=807,2.1 \%)$, annual household income in $2002(n=2,705,7.0 \%)$, gestational age $(n=14,0.04 \%)$, weight at birth $(n=7,0.02 \%)$, and child temperament $(n=374,1.0 \%)$. Finally, 27,870 newborns were included in the analyses ( $72.3 \%$ of the respondents in the 2006 survey wave).

\section{Exposure: paternal childcare}


Paternal childcare was assessed at 18 months of age using the following two measurements: paternal childcare hours on weekdays or weekends, and frequency of each type of caregiving (feeding, changing diapers, bathing, putting the child to sleep, playing with the child at home, and taking the child outside).

Paternal childcare hours were ascertained by asking the following questions: How much time does the father spend with his child on average in one day, except for sleeping hours? Respondents answered in respect of both weekdays and weekends by choosing from the following options: none, $<0.5,0.5-0.9$ hours, 1-1.9 hours, 2-3.9 hours, 4-5.9 hours, and $\geq 6$ hours. Then, we defined the categories, making sure not to highly skew the distribution of each one, into "less than 0.5 hours," "0.5 to 1.9 hours," and " 2 or more hours" for weekdays, and "less than 4 hours," "4 to 5.9 hours," and "6 or more hours" for weekends.

The frequency of each type of paternal caregiving was retrieved using six items: 1) feeding, 2) changing diapers, 3) bathing, 4) putting the child to sleep, 5) playing with the child at home, and 6) taking the child outside. Responses for each question included "not at all," "rarely," "sometimes," and "always" For these categories, we classified "always" and "sometimes" as a high degree of paternal caregiving, and "rarely" and "not at all" as a low degree of paternal caregiving. To calculate the total paternal caregiving scores, each response was scored from 0 to 3 (i.e., "not at all" = 0 and "always" $=3$ ). Thus, the total paternal caregiving scores ranged from 0 to 18 (Cronbach's alpha $=0.77$ ). Paternal caregiving in one type of activity was not strongly correlated with another type of activity (Spearman's correlation coefficients ranging from $0.27-0.50$, Table 1 ). The measurement and the method of specifying the caregiving variable were in line with previous studies $[9,22]$. We divided the total caregiving scores into three groups, namely the "high degree of paternal caregiving" group with more than 1 SD above the mean, the "low degree of paternal caregiving" group with less than 1 SD under the mean, and the "middle degree of paternal caregiving" group.

Table 1. Spearman correlation coefficients between specific types of paternal involvement in caregiving

\begin{tabular}{|lllllll|}
\hline & 1) & 2) & 3) & 4) & 5) & 6) \\
\hline 1) Feeding & 1.00 & & & & & \\
\hline 2) Changing diaper & 0.50 & 1.00 & & & & \\
\hline 3) Bathing & 0.35 & 0.31 & 1.00 & & & \\
\hline 4) Putting the child to sleep & 0.42 & 0.42 & 0.40 & 1.00 & & \\
\hline 5) Playing with the child at home & 0.35 & 0.31 & 0.36 & 0.30 & 1.00 & \\
\hline 6) Taking the child outside & 0.32 & 0.30 & 0.27 & 0.28 & 0.44 & 1.00 \\
\hline
\end{tabular}

\section{Outcome: child behavioral problems}

Child behavioral problems were assessed when the children were 5.5 years old by asking the following six questions which required a yes/no response: 1) Is your child able to listen without fidgeting? 2) Is your child able to focus on a specific task? 3) Is your child patient? 4) Is your child able to express their emotions appropriately? 5) Is your child able to behave in a group situation? 6) Is your child able to keep promises? These variables were developed to identify early signs of behavioral and developmental problems and have been previously used as a set of measurements for behavioral problems [23, 24]. In addition, we defined children with any of these problem behaviors as "children with behavioral problems."

\section{Covariates}

We selected the following variables as potential confounders: the number of siblings, living with grandparent(s), paternal and maternal age at childbirth (< 25 , 25-29, 30-34, 35-39, $\geq 40$ ), paternal and maternal education (lower than high school degree, high school degree, 2-year college or vocational school degree, and a college degree or more), annual household income in 2002 (< JPY 4 million, 4-5.9 million, 6-7.9 million, 8-9.9 million, $\geq 10$ million), maternal childcare hours on weekdays or weekends at 18 months old, and total scores of maternal involvement in caregiving. Maternal involvement in caregiving was scored using the same items used to assess fathers' involvement in caregiving, and the summed score of the six items was used in the analysis.

How fathers are involved with their children is affected by factors relating to the children themselves, such as disabilities and temperament [25, 26]. Therefore, the baseline characteristics of children should also be considered when estimating the effect of paternal childcare: gestational age (<37 weeks, $37-41$ weeks, $\geq 42$ weeks), child's history of hospital admission or visits for congenital diseases, and child's temperament (self-reported dichotomous answers to questions about 18 traits: active, shy of strangers, playful, short-tempered, careful, aggressive, timid, competitive, obedient, independent, stubborn, full of curiosity, fickle, restless, shy, spoiled, easygoing, impatient).

\section{Statistical analysis}

We developed a logistic regression model with odds ratios (ORs) and 95\% confidence intervals (95\% Cls) calculated by adjusting the covariates shown above. In addition to the crude models, two adjusted models were used: Model 1 adjusted for covariates, and Model 2 additionally adjusted for other variables of paternal childcare to examine how each of the paternal childcare variables, that is, paternal childcare hours on weekdays, weekends, and total caregiving scores, affected a child's behavioral problems. We also examined whether the frequency of each type of paternal caregiving was associated with each type of child behavioral problem after adjusting for paternal childcare hours.

All analyses were conducted separately for boys and girls because the effects of paternal childcare may differ due to biologically established sex differences in child development [27]. Analyses were performed using Stata version 15.0 (Stata Corp., College Station, TX, USA).

Page $3 / 14$ 
Approval from the ethics committee of the Tokyo Medical and Dental University was waived because the data were anonymous and available from the Ministry of Health, Labour, and Welfare in Japan upon request. Questionnaire responses from caregivers were considered as informed consent to participate in the study.

\section{Results}

Table 2 shows the characteristics of the respondents in total and stratified by child sex. Preterm births and low birth weights accounted for $4.6 \%$ and $8.1 \%$, respectively. The mean age of the father and mother at the time of birth was $31.7(S D=5.3)$ and $29.7(S D=4.2)$ years, respectively. Half of the families had older siblings when the participating child was born. One of the five families lived with grandparents. The percentage of the history of hospital visits or admissions due to a child's congenital diseases was $2.2 \%$ and $1.0 \%$, respectively. Large differences in proportion were shown between boys and girls in some of the children's temperament traits (e.g., $42.8 \%$ for playful boys compared to $31.1 \%$ for playful girls).

Table 2. Characteristics of the participants 


\begin{tabular}{|c|c|c|c|c|c|c|}
\hline & \multicolumn{2}{|l|}{ Total } & \multicolumn{2}{|l|}{ Boy } & \multicolumn{2}{|l|}{ Girl } \\
\hline & \multicolumn{2}{|c|}{$n=27870$} & \multicolumn{2}{|c|}{$n=14429$} & \multicolumn{2}{|c|}{$\mathrm{n}=13441$} \\
\hline & $\begin{array}{l}\text { No. or } \\
\text { mean }\end{array}$ & $\begin{array}{l}\% \text { or } \\
\text { SD }\end{array}$ & $\begin{array}{l}\text { No. or } \\
\text { mean }\end{array}$ & $\begin{array}{l}\% \text { or } \\
\text { SD }\end{array}$ & $\begin{array}{l}\text { No. or } \\
\text { mean }\end{array}$ & $\%$ or SD \\
\hline \multicolumn{7}{|l|}{ Gestational age (week) } \\
\hline$<37$ & 1277 & 4.6 & 764 & 5.3 & 513 & 3.8 \\
\hline $37-41$ & 26357 & 94.6 & 13542 & 93.9 & 12815 & 95.3 \\
\hline$\geq 42$ & 236 & 0.8 & 123 & 0.9 & 113 & 0.8 \\
\hline \multicolumn{7}{|l|}{ Birth weight (g) } \\
\hline$\geq 2500$ & 25616 & 91.9 & 13384 & 92.8 & 12232 & 91.0 \\
\hline$<2500$ & 2254 & 8.1 & 1045 & 7.2 & 1209 & 9.0 \\
\hline Paternal age at child birth (years old) & 31.7 & 5.3 & 31.8 & 5.3 & 31.7 & 5.3 \\
\hline$<25$ & 1822 & 6.5 & 922 & 6.4 & 900 & 6.7 \\
\hline $25-29$ & 8060 & 28.9 & 4151 & 28.8 & 3909 & 29.1 \\
\hline $30-34$ & 10236 & 36.7 & 5332 & 37.0 & 4904 & 36.5 \\
\hline $35-39$ & 5583 & 20.0 & 2887 & 20.0 & 2696 & 20.1 \\
\hline $40+$ & 2105 & 7.6 & 1100 & 7.6 & 1005 & 7.5 \\
\hline unknown & 64 & 0.2 & 37 & 0.3 & 27 & 0.2 \\
\hline Maternal age at child birth (years old) & 29.7 & 4.2 & 29.7 & 4.2 & 29.7 & 4.2 \\
\hline$<25$ & 2843 & 10.2 & 1465 & 10.2 & 1378 & 10.3 \\
\hline $25-29$ & 11059 & 39.7 & 5747 & 39.8 & 5312 & 39.5 \\
\hline $30-34$ & 10414 & 37.4 & 5402 & 37.4 & 5012 & 37.3 \\
\hline $35-39$ & 3204 & 11.5 & 1629 & 11.3 & 1575 & 11.7 \\
\hline$\geq 40$ & 350 & 1.3 & 186 & 1.3 & 164 & 1.2 \\
\hline \multicolumn{7}{|l|}{ Paternal education } \\
\hline less than high school & 1936 & 6.9 & 1019 & 7.1 & 917 & 6.8 \\
\hline high school & 10773 & 38.7 & 5588 & 38.7 & 5185 & 38.6 \\
\hline some college & 4461 & 16.0 & 2308 & 16.0 & 2153 & 16.0 \\
\hline college or higher & 10700 & 38.4 & 5514 & 38.2 & 5186 & 38.6 \\
\hline \multicolumn{7}{|l|}{ Maternal education } \\
\hline less than high school & 1076 & 3.9 & 539 & 3.7 & 537 & 4.0 \\
\hline high school & 10470 & 37.6 & 5414 & 37.5 & 5056 & 37.6 \\
\hline some college & 12074 & 43.3 & 6330 & 43.9 & 5744 & 42.7 \\
\hline college or higher & 4250 & 15.2 & 2146 & 14.9 & 2104 & 15.7 \\
\hline \multicolumn{7}{|l|}{ Number of siblings } \\
\hline none & 12772 & 45.8 & 6488 & 45.0 & 6284 & 46.8 \\
\hline 1 & 11048 & 39.6 & 5841 & 40.5 & 5207 & 38.7 \\
\hline$\geq 2$ & 4050 & 14.5 & 2100 & 14.6 & 1950 & 14.5 \\
\hline Living with grandparent(s) (answer = yes) & 5866 & 21.0 & 3065 & 21.2 & 2801 & 20.8 \\
\hline \multicolumn{7}{|l|}{ Annual household income (JPY million) } \\
\hline$<4$ & 7966 & 28.6 & 4150 & 28.8 & 3816 & 28.4 \\
\hline $4-5.9$ & 10811 & 38.8 & 5576 & 38.6 & 5235 & 38.9 \\
\hline $6-7.9$ & 5544 & 19.9 & 2845 & 19.7 & 2699 & 20.1 \\
\hline $8-9.9$ & 2099 & 7.5 & 1127 & 7.8 & 972 & 7.2 \\
\hline
\end{tabular}




\begin{tabular}{|c|c|c|c|c|c|c|}
\hline$\geq 10$ & 1450 & 5.2 & 731 & 5.1 & 719 & 5.3 \\
\hline History of hospital visits for child's congenital diseases (answer = yes) & 624 & 2.2 & 344 & 2.4 & 280 & 2.1 \\
\hline $\begin{array}{l}\text { History of hospital admissions for child's congenital diseases (answer = } \\
\text { yes) }\end{array}$ & 267 & 1.0 & 164 & 1.1 & 103 & 0.8 \\
\hline \multicolumn{7}{|l|}{ Child's temperament (answer = yes) } \\
\hline active & 14737 & 52.9 & 7405 & 51.3 & 7332 & 54.5 \\
\hline shy of strangers & 2891 & 10.4 & 1181 & 8.2 & 1710 & 12.7 \\
\hline playful & 10360 & 37.2 & 6182 & 42.8 & 4178 & 31.1 \\
\hline short temper & 4551 & 16.3 & 2445 & 16.9 & 2106 & 15.7 \\
\hline careful & 4172 & 15.0 & 2323 & 16.1 & 1849 & 13.8 \\
\hline aggressive & 12169 & 43.7 & 5254 & 36.4 & 6915 & 51.4 \\
\hline timid & 2084 & 7.5 & 1476 & 10.2 & 608 & 4.5 \\
\hline obedient & 6513 & 23.4 & 3603 & 25.0 & 2910 & 21.7 \\
\hline competitive & 8094 & 29.0 & 3700 & 25.6 & 4394 & 32.7 \\
\hline independent & 12378 & 44.4 & 5465 & 37.9 & 6913 & 51.4 \\
\hline stubborn & 3994 & 14.3 & 2361 & 16.4 & 1633 & 12.1 \\
\hline full of curiosity & 11305 & 40.6 & 5967 & 41.4 & 5338 & 39.7 \\
\hline fickle & 4346 & 15.6 & 2164 & 15.0 & 2182 & 16.2 \\
\hline restless & 6318 & 22.7 & 3845 & 26.6 & 2473 & 18.4 \\
\hline shy & 9400 & 33.7 & 4663 & 32.3 & 4737 & 35.2 \\
\hline spoiled & 15849 & 56.9 & 9150 & 63.4 & 6699 & 49.8 \\
\hline easygoing & 2142 & 7.7 & 1214 & 8.4 & 928 & 6.9 \\
\hline impatient & 1622 & 5.8 & 845 & 5.9 & 777 & 5.8 \\
\hline
\end{tabular}

The distribution of paternal childcare hours and the frequency of each type of caregiving are shown in Table 3. On weekdays, $47.9 \%$ of the fathers spent two or more hours with their children. On weekends, $70.1 \%$ of the fathers spent six or more hours with their children. Little difference was found in these proportions between boys and girls. The total score of paternal caregiving was distributed normally, with a mean of 11.1 and a standard deviation of 3.2. For half of the types of caregiving (i.e., bathing, putting the child to sleep, and taking the child outside), the proportion of fathers who were involved in caregiving "sometimes" or "always," was higher in respect of boys than girls.

Table 3. The distributions of paternal childcare 


\begin{tabular}{|c|c|c|c|c|c|c|c|}
\hline & \multicolumn{2}{|l|}{$\begin{array}{l}\text { Total } \\
n=27870\end{array}$} & \multicolumn{2}{|l|}{$\begin{array}{l}\text { Boy } \\
n=14429\end{array}$} & \multicolumn{2}{|l|}{$\begin{array}{l}\text { Girl } \\
\mathrm{n}=13441\end{array}$} & \multirow[t]{2}{*}{$p$ value* $^{\star}$} \\
\hline & No. or mean & $\%$ or SD & No. or mean & $\%$ or SD & No. or mean & $\%$ or SD & \\
\hline \multicolumn{8}{|l|}{ Childcare hour on weekdays (hour) } \\
\hline$<0.5$ & 4747 & 17.0 & 2456 & 17 & 2291 & 17.0 & 0.89 \\
\hline $0.5-0.9$ & 3654 & 13.1 & 1905 & 13.2 & 1749 & 13.0 & \\
\hline $1-1.9$ & 6104 & 21.9 & 3141 & 21.8 & 2963 & 22.0 & \\
\hline $2-3.9$ & 9432 & 33.8 & 4864 & 33.7 & 4568 & 34.0 & \\
\hline $4-5.9$ & 3348 & 12.0 & 1749 & 12.1 & 1599 & 11.9 & \\
\hline$\geq 6$ & 585 & 2.1 & 314 & 2.2 & 271 & 2.0 & \\
\hline \multicolumn{8}{|l|}{ Childcare hour on weekends (hour) } \\
\hline$<0.5$ & 299 & 1.1 & 158 & 1.1 & 141 & 1.0 & 0.77 \\
\hline $0.5-0.9$ & 470 & 1.7 & 243 & 1.7 & 227 & 1.7 & \\
\hline $1-1.9$ & 1027 & 3.7 & 521 & 3.6 & 506 & 3.8 & \\
\hline $2-3.9$ & 2521 & 9.0 & 1326 & 9.2 & 1195 & 8.9 & \\
\hline $4-5.9$ & 4014 & 14.4 & 2044 & 14.2 & 1970 & 14.7 & \\
\hline$\geq 6$ & 19539 & 70.1 & 10137 & 70.3 & 9402 & 70.0 & \\
\hline Total caregiving score (range: 0 to 18 ) & 11.1 & 3.2 & 11.2 & 3.1 & 11.0 & 3.2 & $<0.001$ \\
\hline \multicolumn{8}{|l|}{ Frequency of feeding } \\
\hline not at all & 3336 & 12.0 & 1733 & 12.0 & 1603 & 11.9 & 0.28 \\
\hline rarely & 7346 & 26.4 & 3738 & 25.9 & 3608 & 26.8 & \\
\hline sometimes & 14593 & 52.4 & 7586 & 52.6 & 7007 & 52.1 & \\
\hline always & 2595 & 9.3 & 1372 & 9.5 & 1223 & 9.1 & \\
\hline \multicolumn{8}{|l|}{ Frequency of changing diaper } \\
\hline not at all & 3416 & 12.3 & 1763 & 12.2 & 1653 & 12.3 & 0.72 \\
\hline rarely & 6571 & 23.6 & 3396 & 23.5 & 3175 & 23.6 & \\
\hline sometimes & 15631 & 56.1 & 8078 & 56.0 & 7553 & 56.2 & \\
\hline always & 2252 & 8.1 & 1192 & 8.3 & 1060 & 7.9 & \\
\hline \multicolumn{8}{|l|}{ Frequency of bathing } \\
\hline not at all & 1337 & 4.8 & 612 & 4.2 & 725 & 5.4 & $<0.001$ \\
\hline rarely & 2612 & 9.4 & 1291 & 8.9 & 1321 & 9.8 & \\
\hline sometimes & 13954 & 50.1 & 7181 & 49.8 & 6773 & 50.4 & \\
\hline always & 9967 & 35.8 & 5345 & 37.0 & 4622 & 34.4 & \\
\hline \multicolumn{8}{|l|}{ Frequency of putting the child to sleep } \\
\hline not at all & 5601 & 20.1 & 2703 & 18.7 & 2898 & 21.6 & $<0.001$ \\
\hline rarely & 8268 & 29.7 & 4218 & 29.2 & 4050 & 30.1 & \\
\hline sometimes & 10950 & 39.3 & 5803 & 40.2 & 5147 & 38.3 & \\
\hline always & 3051 & 10.9 & 1705 & 11.8 & 1346 & 10.0 & \\
\hline \multicolumn{8}{|c|}{ Frequency of playing with the child at home } \\
\hline not at all & 165 & 0.6 & 82 & 0.6 & 83 & 0.6 & 0.61 \\
\hline rarely & 1126 & 4.0 & 582 & 4.0 & 544 & 4.0 & \\
\hline sometimes & 13846 & 49.7 & 7120 & 49.3 & 6726 & 50.0 & \\
\hline always & 12733 & 45.7 & 6645 & 46.1 & 6088 & 45.3 & \\
\hline Frequency of taking the child outside & & & & & & & \\
\hline
\end{tabular}




\begin{tabular}{|lllllll|} 
not at all & 917 & 3.3 & 457 & 3.2 & 460 & 3.4 \\
\hline rarely & 3717 & 13.3 & 1826 & 12.7 & 1891 & 14.1 \\
\hline sometimes & 18882 & 67.8 & 9805 & 68.0 & 9077 & 67.5 \\
\hline always & 4354 & 15.6 & 2341 & 16.2 & 2013 & 15.0 \\
* Chi-square test & & & & & & \\
\hline
\end{tabular}

Table 4 shows the mean of the number of behavioral problems and the percentage of each behavioral problem by sex. Being unable to focus on a specific task was the most observed behavioral problem: $23.9 \%$ of the children. Being unable to express emotions appropriately was the second highest (22.9\%), followed by being unable to keep promises (18.9\%) and being unable to listen without fidgeting (17\%). Boys were more likely to have behavioral problems than girls (1.2 vs. 0.8) and had a significantly higher proportion of behavioral problems than girls, except for "being unable to focus on a specific task."

Table 4. The number of children with behavioral problems

\begin{tabular}{|c|c|c|c|c|c|c|c|}
\hline & $\begin{array}{l}\text { Total } \\
\mathrm{n}=27870\end{array}$ & & $\begin{array}{l}\text { Boy } \\
n=14429\end{array}$ & & $\begin{array}{l}\text { Girl } \\
\mathrm{n}=13441\end{array}$ & & $p$ value $^{\star}$ \\
\hline & mean or No. & SD or $\%$ & mean or No. & SD or $\%$ & mean or No. & SD or $\%$ & \\
\hline Number of behavioral problems & 1.0 & 1.3 & 1.2 & 1.4 & 0.8 & 1.2 & $<0.001$ \\
\hline Any of behavioral problems & 13995 & 50.2 & 7980 & 55.3 & 6015 & 44.8 & $<0.001$ \\
\hline Unable to listen without fidgeting & 4751 & 17.0 & 3131 & 21.7 & 1620 & 12.1 & $<0.001$ \\
\hline Unable to focus on a specific task & 3463 & 12.4 & 1833 & 12.7 & 1630 & 12.1 & 0.15 \\
\hline Unable to be patient & 6656 & 23.9 & 3930 & 27.2 & 2726 & 20.3 & $<0.001$ \\
\hline Unable to express emotions appropriately & 6375 & 22.9 & 3672 & 25.4 & 2703 & 20.1 & $<0.001$ \\
\hline Unable to behave in a group situation & 1656 & 5.9 & 1086 & 7.5 & 570 & 4.2 & $<0.001$ \\
\hline Unable to keep promises & 5270 & 18.9 & 3117 & 21.6 & 2153 & 16.0 & $<0.001$ \\
\hline
\end{tabular}

Table 5 shows the results of the logistic regression analysis to estimate how paternal childcare contributed to behavioral problems in boys and girls. In Model 1, adjusting for covariates, children whose fathers spent two or more hours on weekdays with them had a smaller number of behavioral problems (for boys: OR: $0.70,95 \% \mathrm{Cl}: 0.60$ to 0.82 ; for girls: OR: $0.81,95 \% \mathrm{Cl}: 0.70$ to 0.95 ) as compared with children whose fathers spent less than half an hour on weekdays with them. In addition, children whose fathers spent six or more hours with them on weekends had fewer behavioral problems (for boys: OR: $0.81,95 \% \mathrm{Cl}$ : 0.73 to 0.90 ; for girls: OR: $0.85,95 \% \mathrm{Cl}: 0.76$ to 0.94 ) than children whose fathers spent less than four hours with them on weekends. Children who received a high degree of paternal caregiving, defined as more than $1 \mathrm{SD}$ above the mean of the total paternal caregiving score (i.e., 14.3 points), were observed to have a smaller number of behavioral problems (for boys: OR: $0.76,95 \% \mathrm{Cl}: 0.67$ to 0.88 ; for girls: OR: $0.77,95 \% \mathrm{Cl}$ : 0.67 to 0.89 ) as compared with children who received a low degree of paternal caregiving. When paternal childcare hours and paternal caregiving scores were adjusted simultaneously in Model 2 , the association between paternal childcare hours and problem behaviors remained in boys (for weekdays: OR: $0.71,95 \% \mathrm{Cl}$ : 0.60 to 0.84 ; for weekends: $0.86,95 \%$ Cl: 0.76 to 0.96 in Table 5), but there was no significant association between total caregiving score and problem behaviors in boys. For girls, the association between paternal childcare hours on weekdays and problem behaviors remained (OR: $0.83,95 \% \mathrm{Cl}: 0.710 .99)$. Paternal childcare hours on weekends were protective against problem behaviors in girls, although the difference was not statistically significant (OR: $0.89,95 \%$ Cl: 0.79 to 1.00$)$. There was no significant multicollinearity among the variables adjusted for in Model 2.

Table 5. The effects of paternal childcare-hour and caregiving score on behavioral problems in 5.5 year-old children; result of the logistic regression model 


\begin{tabular}{|c|c|c|c|c|c|c|c|c|c|}
\hline & \multicolumn{3}{|c|}{ Crude model } & \multicolumn{3}{|c|}{ Model 1} & \multicolumn{3}{|c|}{ Model 2} \\
\hline & $\mathrm{OR}$ & \multicolumn{2}{|c|}{$95 \% \mathrm{Cl}$} & OR & \multicolumn{2}{|l|}{$95 \% \mathrm{Cl}$} & OR & \multicolumn{2}{|c|}{$95 \% \mathrm{Cl}$} \\
\hline \multicolumn{10}{|l|}{ Boys } \\
\hline \multicolumn{10}{|c|}{ Childcare-hour on weekdays (ref. $<0.5$ hours) } \\
\hline 0.5 to 1.9 hours & 0.91 & 0.78 & 1.05 & 0.82 & 0.70 & 0.96 & 0.82 & 0.70 & 0.96 \\
\hline \multirow[t]{2}{*}{$\geq 2$ hours } & 0.87 & 0.75 & 1.01 & 0.70 & 0.60 & 0.82 & 0.71 & 0.60 & 0.84 \\
\hline & \multicolumn{3}{|c|}{ ( $p$ for trend) 0.07} & \multicolumn{3}{|c|}{$(p$ for trend $)<0.001$} & \multicolumn{3}{|c|}{$(p$ for trend $)<0.001$} \\
\hline \multicolumn{10}{|c|}{ Childcare-hour on weekends (ref. $<4$ hours) } \\
\hline 4 to 5.9 hours & 0.93 & 0.82 & 1.05 & 0.92 & 0.81 & 1.05 & 0.96 & 0.84 & 1.10 \\
\hline \multirow[t]{2}{*}{$\geq 6$ hours } & 0.79 & 0.72 & 0.87 & 0.81 & 0.73 & 0.90 & 0.86 & 0.76 & 0.96 \\
\hline & \multicolumn{3}{|c|}{$(p$ for trend $)<0.001$} & \multicolumn{3}{|c|}{$(p$ for trend $)<0.001$} & \multicolumn{3}{|c|}{ ( $p$ for trend) 0.004} \\
\hline \multicolumn{10}{|c|}{ Total caregiving score (ref. low) } \\
\hline middle & 0.92 & 0.83 & 1.02 & 0.87 & 0.78 & 0.97 & 0.98 & 0.87 & 1.10 \\
\hline \multirow[t]{2}{*}{ high } & 0.86 & 0.76 & 0.98 & 0.76 & 0.67 & 0.88 & 0.92 & 0.79 & 1.07 \\
\hline & \multicolumn{3}{|c|}{ ( $p$ for trend) 0.03} & \multicolumn{3}{|c|}{$(p$ for trend $)<0.001$} & \multicolumn{3}{|c|}{ ( $p$ for trend) 0.29} \\
\hline \multicolumn{10}{|l|}{ Girls } \\
\hline \multicolumn{10}{|c|}{ Childcare-hour on weekdays (ref. $<0.5$ hours) } \\
\hline 0.5 to 1.9 hours & 0.94 & 0.81 & 1.09 & 0.90 & 0.77 & 1.05 & 0.91 & 0.78 & 1.06 \\
\hline$\geq 2$ hours & 0.95 & 0.82 & 1.11 & 0.81 & 0.70 & 0.95 & 0.83 & 0.71 & 0.99 \\
\hline$p$ for trend & \multicolumn{3}{|c|}{ ( $p$ for trend) } & \multicolumn{3}{|c|}{ ( $p$ for trend) 0.001} & \multicolumn{3}{|c|}{ ( $p$ for trend) 0.02} \\
\hline \multicolumn{10}{|c|}{ Childcare-hour on weekends (ref. $<4$ hours) } \\
\hline 4 to 5.9 hours & 0.99 & 0.88 & 1.12 & 1.00 & 0.88 & 1.14 & 1.05 & 0.91 & 1.20 \\
\hline$\geq 6$ hours & 0.79 & 0.72 & 0.87 & 0.85 & 0.76 & 0.94 & 0.89 & 0.79 & 1.00 \\
\hline$p$ for trend & \multicolumn{3}{|c|}{$(p$ for trend $)<0.001$} & \multicolumn{3}{|c|}{$(p$ for trend $)<0.001$} & ( $p$ for & nd) 0.0 & \\
\hline Total caregiving & & & & & & & & & \\
\hline middle & 0.91 & 0.82 & 1.00 & 0.85 & 0.76 & 0.94 & 0.91 & 0.81 & 1.01 \\
\hline high & 0.90 & 0.78 & 1.02 & 0.77 & 0.67 & 0.89 & 0.88 & 0.75 & 1.03 \\
\hline$p$ for trend & (p for & nd) 0.10 & & $(p$ for $t$ & nd) $<0.0$ & & $(p$ for & nd) 0.1 & \\
\hline $\begin{array}{l}\text { a) Model } 1 \text { adjus } \\
\text { education, annu } \\
\text { in caregiving, ge } \\
\text { b) Model } 2 \text { adjus } \\
\text { c) Bold values de }\end{array}$ & $\begin{array}{l}\text { ving wi } \\
\text { on wee } \\
\text { ion or } \\
\text { I childc }\end{array}$ & $\begin{array}{l}\text { grandpa } \\
\text { ays or } w \\
\text { ts for co }\end{array}$ & $\begin{array}{l}\text { t(s), pa } \\
\text { kends } \\
\text { enital }\end{array}$ & $\begin{array}{l}\text { nal and } \\
8 \text { month } \\
\text { ases, ch }\end{array}$ & $\begin{array}{l}\text { aternal a } \\
\text { old, and } \\
\text { 's tempe }\end{array}$ & $\begin{array}{l}\text { at chil } \\
\text { e totals } \\
\text { ment). }\end{array}$ & $\begin{array}{l}\text { rth, pat } \\
\text { es of } n\end{array}$ & $\begin{array}{l}\text { al and } \\
\text { ternal in }\end{array}$ & $\begin{array}{l}\text { ernal } \\
\text { vemen }\end{array}$ \\
\hline
\end{tabular}

Several paternal caregiving behaviors decreased specific problem behaviors in children, as shown in Tables 6 and 7 . For example, boys whose fathers always fed them had fewer behavioral problems, such as not listening without fidgeting (OR: $0.75,95 \% \mathrm{Cl}: 0.61$ to 0.90 ), not being patient (OR: $0.76,95 \% \mathrm{Cl}$ : 0.63 to 0.90), and not keeping promises (OR: $0.82,95 \% \mathrm{Cl}: 0.68$ to 0.99 , Table 6 ). In addition, boys whose fathers always changed their diapers had fewer behavioral problems such as not listening without fidgeting (OR: $0.73,95 \% \mathrm{Cl}: 0.60$ to 0.90$)$, not focusing on a specific task (OR: 0.75, 95\% Cl: 058. 0.96), and not expressing their emotions appropriately (OR: $0.81,95 \% \mathrm{Cl}: 0.670 .98)$. Paternal childcare, such as playing with their children at home or taking their children outside, had a protective dose-effect on most behavioral problems in boys at age 5.5 (all p for trend < 0.05 , except for expressing emotions appropriately).

Table 6. The effects of paternal childcare on behavioral problems in 5.5 year-old boys; result of the logistic regression model 


\begin{tabular}{|c|c|c|c|c|c|c|c|c|c|c|c|c|}
\hline \multicolumn{2}{|c|}{ Behavioral problem } & \multicolumn{2}{|c|}{$\begin{array}{l}\text { Unable to listen } \\
\text { without fidgeting }\end{array}$} & \multicolumn{2}{|c|}{$\begin{array}{l}\text { Unable to focus on } \\
\text { a specific task }\end{array}$} & \multicolumn{2}{|c|}{ Unable to be patient } & \multicolumn{2}{|c|}{$\begin{array}{l}\text { Unable to express } \\
\text { emotions } \\
\text { appropriately }\end{array}$} & \multicolumn{2}{|c|}{$\begin{array}{l}\text { Unable to behave in } \\
\text { a group situation }\end{array}$} & $\begin{array}{l}U \\
\mathrm{p}\end{array}$ \\
\hline OR & $95 \% \mathrm{Cl}$ & OR & $95 \% \mathrm{Cl}$ & OR & $95 \% \mathrm{Cl}$ & OR & $95 \% \mathrm{Cl}$ & OR & $95 \% \mathrm{Cl}$ & OR & $95 \% \mathrm{Cl}$ & $\mathrm{C}$ \\
\hline
\end{tabular}

Frequency of feeding (ref.

not at all)

\begin{tabular}{|c|c|c|c|c|c|c|c|c|c|c|c|c|c|c|c|c|c|c|c|}
\hline rarely & 0.85 & 0.76 & 0.96 & 0.80 & 0.69 & 0.93 & 0.99 & 0.83 & 1.19 & 0.81 & 0.71 & 0.93 & 0.88 & 0.77 & 1.01 & 0.89 & 0.72 & 1.10 & 0 \\
\hline sometimes & 0.86 & 0.77 & 0.97 & 0.86 & 0.75 & 0.98 & 1.02 & 0.86 & 1.21 & 0.82 & 0.72 & 0.93 & 0.88 & 0.78 & 1.00 & 0.90 & 0.74 & 1.10 & 0 \\
\hline always & 0.85 & 0.73 & 1.00 & 0.75 & 0.61 & 0.90 & 0.87 & 0.69 & 1.11 & 0.76 & 0.63 & 0.90 & 0.87 & 0.73 & 1.04 & 0.91 & 0.68 & 1.21 & 0 \\
\hline
\end{tabular}

Frequency of changing diaper (ref.

not at all)

\begin{tabular}{|c|c|c|c|c|c|c|c|c|c|c|c|c|c|c|c|c|c|c|c|}
\hline rarely & 0.90 & 0.80 & 1.02 & 0.84 & 0.72 & 0.97 & 0.87 & 0.73 & 1.04 & 0.90 & 0.79 & 1.03 & 0.97 & 0.84 & 1.11 & 0.99 & 0.79 & 1.24 & 0 \\
\hline sometimes & 0.94 & 0.84 & 1.05 & 0.84 & 0.73 & 0.96 & 0.95 & 0.81 & 1.13 & 0.96 & 0.85 & 1.09 & 0.89 & 0.79 & 1.02 & 1.07 & 0.87 & 1.32 & 1 \\
\hline always & 0.82 & 0.70 & 0.97 & 0.73 & 0.60 & 0.90 & 0.75 & 0.58 & 0.96 & 0.88 & 0.73 & 1.06 & 0.81 & 0.67 & 0.98 & 1.15 & 0.85 & 1.55 & 0 \\
\hline
\end{tabular}

Frequency of bathing (ref.

not at all)

\begin{tabular}{|c|c|c|c|c|c|c|c|c|c|c|c|c|c|c|c|c|c|c|c|}
\hline rarely & 0.92 & 0.75 & 1.12 & 1.16 & 0.91 & 1.49 & 0.89 & 0.66 & 1.19 & 1.17 & 0.94 & 1.46 & 0.80 & 0.64 & 1.01 & 0.92 & 0.65 & 1.30 & 1 \\
\hline sometimes & 0.99 & 0.83 & 1.19 & 1.07 & 0.87 & 1.33 & 0.95 & 0.74 & 1.23 & 1.01 & 0.83 & 1.23 & 0.88 & 0.72 & 1.06 & 0.79 & 0.59 & 1.07 & 1 \\
\hline always & 0.88 & 0.73 & 1.07 & 0.98 & 0.78 & 1.23 & 0.81 & 0.62 & 1.05 & 0.94 & 0.76 & 1.15 & 0.78 & 0.64 & 0.96 & 0.81 & 0.59 & 1.10 & 1 \\
\hline
\end{tabular}

Frequency of putting the child to sleep (ref. not at

all)

\begin{tabular}{|c|c|c|c|c|c|c|c|c|c|c|c|c|c|c|c|c|c|c|}
\hline rarely & 0.99 & 0.89 & 1.10 & 1.01 & 0.89 & 1.15 & 0.92 & 0.79 & 1.07 & 0.93 & 0.83 & 1.04 & 0.91 & 0.81 & 1.02 & 0.88 & 0.73 & 1.06 \\
\hline sometimes & 0.97 & 0.88 & 1.07 & 1.06 & 0.94 & 1.20 & 1.05 & 0.90 & 1.22 & 0.93 & 0.83 & 1.04 & 0.88 & 0.78 & 0.98 & 0.92 & 0.76 & 1.10 \\
\hline always & 1.05 & 0.91 & 1.20 & 1.07 & 0.91 & 1.27 & 0.90 & 0.73 & 1.10 & 0.89 & 0.77 & 1.04 & 0.95 & 0.82 & 1.11 & 1.15 & 0.91 & 1.46 \\
\hline
\end{tabular}

Frequency of playing with the child at home (ref.

not at all)

\begin{tabular}{|c|c|c|c|c|c|c|c|c|c|c|c|c|c|c|c|c|c|c|}
\hline rarely & 1.16 & 0.70 & 1.89 & 1.06 & 0.61 & 1.85 & 0.96 & 0.48 & 1.92 & 0.97 & 0.58 & 1.62 & 0.75 & 0.45 & 1.25 & 0.79 & 0.39 & 1.57 \\
\hline sometimes & 1.07 & 0.67 & 1.70 & 0.73 & 0.43 & 1.24 & 0.82 & 0.42 & 1.58 & 0.86 & 0.53 & 1.40 & 0.78 & 0.48 & 1.26 & 0.56 & 0.29 & 1.07 \\
\hline always & 0.95 & 0.59 & 1.52 & 0.65 & 0.38 & 1.11 & 0.75 & 0.39 & 1.46 & 0.79 & 0.48 & 1.30 & 0.65 & 0.40 & 1.07 & 0.51 & 0.26 & 0.99 \\
\hline
\end{tabular}

Frequency of taking the child outside (ref.

not at all)

\begin{tabular}{|c|c|c|c|c|c|c|c|c|c|c|c|c|c|c|c|c|c|c|c|}
\hline rarely & 1.05 & 0.84 & 1.31 & 0.85 & 0.66 & 1.10 & 1.03 & 0.76 & 1.41 & 0.91 & 0.72 & 1.15 & 0.91 & 0.71 & 1.15 & 0.88 & 0.62 & 1.25 & 1 \\
\hline sometimes & 0.92 & 0.75 & 1.13 & 0.75 & 0.60 & 0.95 & 0.86 & 0.64 & 1.15 & 0.80 & 0.65 & 1.00 & 0.89 & 0.71 & 1.11 & 0.76 & 0.54 & 1.05 & 0 \\
\hline \multirow[t]{2}{*}{ always } & 0.89 & 0.71 & 1.11 & 0.78 & 0.60 & 1.01 & 0.84 & 0.61 & 1.15 & 0.78 & 0.62 & 0.99 & 0.82 & 0.64 & 1.04 & 0.73 & 0.51 & 1.06 & 0 \\
\hline & \multicolumn{3}{|c|}{ ( $p$ for trend) 0.02} & \multicolumn{3}{|c|}{ ( $p$ for trend) 0.05} & \multicolumn{3}{|c|}{ ( $p$ for trend) 0.04} & \multicolumn{3}{|c|}{ ( $p$ for trend) 0.01} & \multicolumn{3}{|c|}{ ( $p$ for trend) 0.09} & \multicolumn{3}{|c|}{ ( $p$ for trend) 0.05} & $(1$ \\
\hline
\end{tabular}

a) Adjusted covariates (number of siblings, living with grandparent(s), paternal and maternal age at child birth, paternal and maternal education, annual hous maternal childcare hours on weekdays or weekends at 18 months old, and the total scores of maternal involvement in caregiving, gestational age, child's hist admission or visits for congenital diseases, child's temperament), and parental spending hours with children.

b) Bold values denote statistical significance at the $\mathrm{p}<0.05$.

Girls whose fathers always fed them or changed their diapers had fewer problems with lacking patience (OR: 0.74, $95 \%$ Cl: 0.60 to $0.91,0 R: 0.74,95 \% \mathrm{Cl}: 0.60$ to 0.91 ) (Table 7). Paternal playing at home with their children had a protective dose-effect on all types of problem behaviors in girls at age 5 (all p for trend < 0.05). Interestingly, girls whose fathers sometimes put them to sleep had more problem behaviors, such as not listening without fidgeting (OR: $1.17,95 \% \mathrm{Cl}$ : 1.00 to 0.37 ) and not expressing their emotions appropriately (OR: $1.14,95 \% \mathrm{Cl}: 1.01$ to 1.30 ) than girls whose fathers never put them to sleep.

Table 7. The effects of paternal childcare on behavioral problems in 5.5 year-old girls; result of the logistic regression model 


\begin{tabular}{|c|c|c|c|c|c|c|c|c|c|c|c|c|}
\hline \multicolumn{2}{|c|}{ Behavioral problem } & \multicolumn{2}{|c|}{$\begin{array}{l}\text { Unable to listen } \\
\text { without fidgeting }\end{array}$} & \multicolumn{2}{|c|}{$\begin{array}{l}\text { Unable to focus on } \\
\text { a specific task }\end{array}$} & \multicolumn{2}{|c|}{ Unable to be patient } & \multicolumn{2}{|c|}{$\begin{array}{l}\text { Unable to express } \\
\text { emotions } \\
\text { appropriately }\end{array}$} & \multicolumn{2}{|c|}{$\begin{array}{l}\text { Unable to behave in } \\
\text { a group situation }\end{array}$} & $\begin{array}{l}\mathrm{U} \\
\mathrm{p}\end{array}$ \\
\hline OR & $95 \% \mathrm{Cl}$ & OR & $95 \% \mathrm{Cl}$ & OR & $95 \% \mathrm{Cl}$ & OR & $95 \% \mathrm{Cl}$ & OR & $95 \% \mathrm{Cl}$ & OR & $95 \% \mathrm{Cl}$ & $\mathrm{C}$ \\
\hline
\end{tabular}

Frequency of feeding (ref.

not at all)

\begin{tabular}{|c|c|c|c|c|c|c|c|c|c|c|c|c|c|c|c|c|c|c|c|}
\hline rarely & 1.05 & 0.92 & 1.19 & 0.96 & 0.79 & 1.16 & 0.96 & 0.79 & 1.15 & 0.96 & 0.82 & 1.11 & 1.02 & 0.88 & 1.19 & 0.87 & 0.65 & 1.15 & 1 \\
\hline sometimes & 0.94 & 0.84 & 1.06 & 0.96 & 0.80 & 1.16 & 0.86 & 0.72 & 1.03 & 0.87 & 0.76 & 1.01 & 0.96 & 0.83 & 1.11 & 0.89 & 0.68 & 1.16 & 1 \\
\hline \multirow[t]{2}{*}{ always } & 1.01 & 0.85 & 1.19 & 0.85 & 0.66 & 1.10 & 0.97 & 0.76 & 1.25 & 0.74 & 0.60 & 0.91 & 1.06 & 0.86 & 1.29 & 1.10 & 0.75 & 1.61 & 1 \\
\hline & \multicolumn{3}{|c|}{ ( $p$ for trend) 0.23} & \multicolumn{3}{|c|}{ ( $p$ for trend) 0.38} & \multicolumn{3}{|c|}{ ( $p$ for trend) 0.22} & \multicolumn{3}{|c|}{ ( $p$ for trend) 0.002} & \multicolumn{3}{|c|}{ ( $p$ for trend) 0.79} & \multicolumn{3}{|c|}{ ( $p$ for trend) 0.92} & (1 \\
\hline
\end{tabular}

Frequency of changing diaper (ref.

not at all)

\begin{tabular}{llllllllllllllllllll} 
rarely & 0.99 & 0.88 & 1.13 & 0.95 & 0.78 & 1.15 & 0.91 & 0.76 & 1.11 & 0.87 & 0.75 & 1.01 & 1.07 & 0.91 & 1.24 & 1.12 & 0.84 & 1.50 & 1 \\
\hline sometimes & 0.93 & 0.83 & 1.05 & 0.90 & 0.76 & 1.08 & 0.96 & 0.80 & 1.14 & $\mathbf{0 . 8 5}$ & $\mathbf{0 . 7 4}$ & $\mathbf{0 . 9 8}$ & 0.98 & 0.85 & 1.13 & 1.00 & 0.76 & 1.33 & 0 \\
always & 0.97 & 0.82 & 1.15 & 0.88 & 0.68 & 1.14 & 0.97 & 0.75 & 1.26 & $\mathbf{0 . 7 4}$ & $\mathbf{0 . 6 0}$ & $\mathbf{0 . 9 1}$ & 1.15 & 0.93 & 1.42 & 1.08 & 0.71 & 1.64 & 1 \\
\hline & & $(p$ for trend) & 0.22 & &
\end{tabular}

Frequency of bathing (ref.

not at all)

\begin{tabular}{|c|c|c|c|c|c|c|c|c|c|c|c|c|c|c|c|c|c|c|c|}
\hline rarely & 0.95 & 0.79 & 1.15 & 1.11 & 0.84 & 1.48 & 1.02 & 0.77 & 1.35 & 0.91 & 0.73 & 1.15 & 0.95 & 0.76 & 1.19 & 1.09 & 0.72 & 1.63 & 0 \\
\hline sometimes & 0.91 & 0.77 & 1.07 & 0.86 & 0.67 & 1.10 & 0.87 & 0.68 & 1.10 & 0.92 & 0.76 & 1.12 & 0.93 & 0.77 & 1.13 & 0.81 & 0.57 & 1.17 & 0 \\
\hline \multirow[t]{2}{*}{ always } & 0.88 & 0.74 & 1.05 & 0.82 & 0.63 & 1.07 & 0.88 & 0.68 & 1.14 & 0.92 & 0.75 & 1.14 & 0.92 & 0.75 & 1.14 & 0.76 & 0.52 & 1.12 & 0 \\
\hline & \multicolumn{3}{|c|}{ ( $p$ for trend) 0.13} & \multicolumn{3}{|c|}{ ( $p$ for trend) 0.01} & \multicolumn{3}{|c|}{ ( $p$ for trend) 0.19} & \multicolumn{3}{|c|}{ ( $p$ for trend) 0.65} & \multicolumn{3}{|c|}{ ( $p$ for trend) 0.48} & \multicolumn{3}{|c|}{ ( $p$ for trend) 0.03} & (1) \\
\hline
\end{tabular}

Frequency of putting the child to sleep

(ref. not at all)

\begin{tabular}{|c|c|c|c|c|c|c|c|c|c|c|c|c|c|c|c|c|c|c|c|}
\hline rarely & 1.01 & 0.91 & 1.12 & 1.01 & 0.86 & 1.18 & 0.93 & 0.80 & 1.08 & 0.97 & 0.85 & 1.09 & 1.10 & 0.97 & 1.24 & 1.01 & 0.80 & 1.28 & 0 \\
\hline sometimes & 1.00 & 0.91 & 1.11 & 1.17 & 1.00 & 1.37 & 0.88 & 0.75 & 1.02 & 0.93 & 0.82 & 1.05 & 1.14 & 1.01 & 1.30 & 1.02 & 0.80 & 1.29 & 0 \\
\hline \multirow[t]{2}{*}{ always } & 0.88 & 0.76 & 1.03 & 0.97 & 0.77 & 1.21 & 0.89 & 0.72 & 1.11 & 0.90 & 0.75 & 1.08 & 1.08 & 0.90 & 1.29 & 0.80 & 0.55 & 1.15 & 0 \\
\hline & \multicolumn{3}{|c|}{ ( $p$ for trend) 0.96} & \multicolumn{3}{|c|}{ ( $p$ for trend) 0.08} & \multicolumn{3}{|c|}{ ( $p$ for trend) 0.12} & \multicolumn{3}{|c|}{ ( $p$ for trend) 0.15} & \multicolumn{3}{|c|}{ ( $p$ for trend) 0.12} & \multicolumn{3}{|c|}{ ( $p$ for trend) 0.80} & (1 \\
\hline
\end{tabular}

Frequency of playing with the child at home (ref.

not at all)

\begin{tabular}{|c|c|c|c|c|c|c|c|c|c|c|c|c|c|c|c|c|c|c|c|}
\hline rarely & 0.88 & 0.54 & 1.43 & 0.95 & 0.48 & 1.89 & 0.85 & 0.44 & 1.64 & 0.63 & 0.38 & 1.06 & 1.28 & 0.70 & 2.33 & 0.89 & 0.36 & 2.23 & 0 \\
\hline sometimes & 0.86 & 0.54 & 1.35 & 0.75 & 0.39 & 1.44 & 0.72 & 0.39 & 1.33 & 0.54 & 0.33 & 0.88 & 1.10 & 0.62 & 1.96 & 0.65 & 0.27 & 1.55 & 0 \\
\hline always & 0.75 & 0.47 & 1.20 & 0.64 & 0.33 & 1.25 & 0.66 & 0.36 & 1.24 & 0.48 & 0.29 & 0.78 & 0.96 & 0.54 & 1.71 & 0.56 & 0.23 & 1.36 & 0 \\
\hline
\end{tabular}

Frequency of taking the child outside (ref.

not at all)

\begin{tabular}{|c|c|c|c|c|c|c|c|c|c|c|c|c|c|c|c|c|c|c|c|}
\hline rarely & 0.78 & 0.63 & 0.97 & 0.85 & 0.62 & 1.17 & 0.98 & 0.71 & 1.36 & 0.84 & 0.65 & 1.09 & 0.90 & 0.70 & 1.16 & 0.76 & 0.49 & 1.17 & 0 \\
\hline sometimes & 0.75 & 0.61 & 0.92 & 0.82 & 0.61 & 1.11 & 0.95 & 0.70 & 1.29 & 0.89 & 0.70 & 1.13 & 0.83 & 0.65 & 1.05 & 0.69 & 0.46 & 1.04 & 0 \\
\hline \multirow[t]{2}{*}{ always } & 0.71 & 0.57 & 0.89 & 0.79 & 0.57 & 1.11 & 0.90 & 0.64 & 1.26 & 0.82 & 0.63 & 1.07 & 0.77 & 0.59 & 1.00 & 0.73 & 0.46 & 1.16 & 0 \\
\hline & \multicolumn{3}{|c|}{ ( $p$ for trend) 0.01} & \multicolumn{3}{|c|}{ ( $p$ for trend) 0.24} & \multicolumn{3}{|c|}{ ( $p$ for trend) 0.38} & \multicolumn{3}{|c|}{ ( $p$ for trend) 0.40} & \multicolumn{3}{|c|}{ ( $p$ for trend) 0.02} & \multicolumn{3}{|c|}{ ( $p$ for trend) 0.24} & () \\
\hline
\end{tabular}

a) Adjusted covariates (number of siblings, living with grandparent(s), paternal and maternal age at child birth, paternal and maternal education, annual hous maternal childcare hours on weekdays or weekends at 18 months old, and the total scores of maternal involvement in caregiving, gestational age, child's hist admission or visits for congenital diseases, child's temperament), and parental spending hours with children.

b) Bold values denote statistical significance at the $p<0.05$.

\section{Discussion}

The present study examined the association between paternal childcare during infancy and behavioral problems at 5.5 years of age using a Japanese nationwide population-based longitudinal cohort. Our findings suggest that longer paternal childcare hours in the infancy period were associated with a lower risk of behavioral problems at 5.5 years of age, even after adjusting for paternal parenting behaviors in infancy. We also found that several paternal caregiving behaviors showed a strong preventive effect on specific problem behaviors in children, such as taking the child outside for boys and playing at home for both boys and girls. 
The protective effect of paternal involvement in childcare on children's subsequent behavioral problems is consistent with previous studies that reported that child appropriate behavior was positively associated with parenting by the father, after adjusting for other confounders, including parenting by the mother [ 12 , $13,28,29]$. The current study also revealed the effect of paternal time spent with children on both weekdays and weekends on their subsequent behavior. On the other hand, the overall frequency of paternal childcare during infancy was not independently associated with behavioral problems in children at 5.5 years of age, after adjusting for the amount of time fathers spent with their children, suggesting that the duration, but not the quality of paternal involvement, matters for children's subsequent behavioral problems. Previous studies have reported that fathers' parenting quality, as well as the quantity of routine care provided, are associated with a lower risk of child behavioral problems [12, 28]. In addition, fathers who spend more time on child caregiving have a higher quality of interaction with their children, because primary caregiving fathers have a better understanding of how to care for and play with their children as a result of the greater time they have spent with them [30]. This study did not directly measure the quality of parenting; however, spending more time with children on weekdays and weekends could improve paternal caregiving skills, which may have a positive impact on children's behavior.

Children whose fathers were always involved in some type of essential childcare, such as feeding and changing diapers, had fewer problems in specific behaviors. Possibly, paternal childcare, which is essential for a child's life, might help to reduce mothers' childcare burdens and contribute to lower maternal stress, which is considered as a risk for behavioral problems in children [31, 32]. On the other hand, playing with children at home or taking them outside is a more complex type of interaction between fathers and children, which is an important stimulus for the child's socioemotional development [33]. Therefore, in this study, we found that these types of paternal caregiving could have preventive effects on a variety of problem behaviors in children.

We estimated the sex-specific effects of paternal childcare on behavioral problems in children. Our results did not confirm the sex difference in paternal time spent with the child on weekdays and weekends. Fathers of boys, however, were involved slightly more frequently than those of girls in several types of caregiving, such as bathing, putting their children to sleep, and taking them outside. These sex differences in paternal involvement have been found in previous studies and are considered to be induced in response to underlying biological or psychosocial differences in children [27, 34]. Moreover, the influence of paternal caregiving on the child's behavior differed depending on the child's sex in this study. For example, boys showed reduced behavioral problems when their fathers often took them outside, while girls showed reduced behavioral problems when their fathers often played with them at home. The reason for these sex-specific effects might be explained by the well-established gender differences in behavior [34-36]; however, further research is required.

This study has several strengths. First, in our analysis, we considered the child's original temperament and congenital disabilities, which could be related to the involvement of fathers in parenting as well as behavioral problems in their children. Therefore, the present study provides robust evidence that paternal childcare has a positive effect on children's behavioral problems. Second, these results were obtained from a representative sample in Japan, in which fathers typically spend long hours commuting and working and, therefore, spend less time with their children than fathers in Western countries [37, 38]. This study provided a test of the generalizability of the hypothesis that paternal childcare would have a protective effect on child behavior. Third, we used a prospective cohort study; thus, reverse causation was unlikely.

Despite its strengths, this study also has some limitations. First, we used maternal reports to evaluate paternal childcare because of the lack of precise measures. In addition, our survey did not ask about the actual amount of time spent on each type of paternal caregiving behavior. According to previous studies, mothers' evaluations of paternal childcare are susceptible to influence by marital satisfaction and maternal emotional well-being [39, 40]. Other objective measures of paternal childcare should be used in future studies. Second, the outcome in this study, that is, behavioral problems in 5.5-year-old children, were also self-reported by their mothers. Mothers may be one of the primary caregivers for children in most cases; however, they may not be able to fully observe their child's behavior in situations other than at home. Third, our results could still be biased by residual confounding that we could not measure in this survey, such as marital relationships or paternal mental health. Data about the father's health or relationships with their partners are scarce, so their potential effects on paternal childcare and child development are not widely recognized [41]. Researchers and practitioners would need to take these family factors into account when considering the interactions between fathers and children.

\section{Conclusions}

Despite these limitations, the present study suggests that paternal childcare in the infancy period could prevent subsequent behavioral problems in children based on a national prospective study in Japan. Our study also showed that several specific caregiving behaviors by fathers, such as playing with their children at home or taking them outside, may play an important role in appropriate behavioral development in early childhood, which depends on the child's sex. Promoting paternal childcare support would augment the quantity and quality of paternal caregiving, which in turn could have a beneficial effect on child behavioral development. The policies that support child-rearing fathers, including the promotion of work environments that facilitate paid parental leave, restriction of overtime work, and incentives for remote or flex-time work, could be linked to the prevention of behavioral problems in children. Further studies using more detailed data on paternal childcare are required to elucidate the mechanisms by which fathers' involvement in childcare could have a protective effect on early childhood behavior.

\section{Abbreviations}

OR: odds ratio; Cl: confidence interval; SD: standard deviation

\section{Declarations}

Ethics approval and consent to participate: All procedures were conducted in accordance with the Declaration of Helsinki and Ethical Guidelines for Medical and Health Research Involving Human Subjects. Approval from the ethics committee of the Tokyo Medical and Dental University was waived because the data 
were anonymous and available from the Ministry of Health, Labour, and Welfare in Japan upon request. Questionnaire responses from participants were considered as informed consent to participate in the study.

Consent for publication: Not applicable.

Availability of data and materials: The data that support the findings of this study are available from the Ministry of Health, Labor, and Welfare in Japan but restrictions apply to the availability of these data, which were used under license for the current study, and so are not publicly available.

Competing interests: The authors declare that they have no competing interests.

Funding: Grants-in-Aid for Scientific Research from the Japan Society for the Promotion of Science (JSPS KAKENHI Grant Number 16H03276).

Authors' contributions: MO conceptualized and designed the study, coordinated the study, analyzed data, drafted the initial manuscript, and reviewed and revised the manuscript. TF conceptualized and designed the study, coordinated and supervised the study, reviewed and revised the manuscript. All authors approved the final manuscript as submitted and agree to be accountable for all aspects of the work.

Acknowledgements: Not applicable.

\section{References}

1. Lamb ME: The role of the father in child development. New York: John Wiley \& Sons; 2004.

2. Yogman MW, Kindlon D, Earls F: Father involvement and cognitive-behavioral outcomes of preterm infants. J Am Acad Child Adolesc Psychiatry 1995 , 34(1):58-66.

3. Tamis-LeMonda CS, Shannon JD, Cabrera NJ, Lamb ME: Fathers and mothers at play with their 2-and 3-year-olds: contributions to language and cognitive development. Child Dev 2004, 75(6):1806-1820.

4. Black MM, Dubowitz H, Starr RH: African American fathers in low income, urban families: development, behavior, and home environment of their threeyear-old children. Child Dev 1999, 70(4):967-978.

5. Abate KH, Belachew T: Women's autonomy and men's involvement in child care and feeding as predictors of infant and young child anthropometric indices in coffee farming households of Jimma Zone, South West of Ethiopia. PLoS One 2017, 12(3):e0172885.

6. Lindsey EW, Cremeens PR, Caldera YM: Mother-child and father-child mutuality in two contexts: consequences for young children's peer relationships. Infant Child Dev 2010, 19(2):142-160.

7. King V: Nonresident father involvement and child well-being - can dads make a difference. Journal of Family Issues 1994, 15(1):78-96.

8. Flouri E, Buchanan A: Early father's and mother's involvement and child's later educational outcomes. Br J Educ Psycho/ $2004,74: 141-153$.

9. Fujiwara T, Okuyama M, Takahashi K: Patemal involvement in childcare and unintentional injury of young children: a population-based cohort study in Japan. International Journal of Epidemiology 2010, 39(2):588-597.

10. Amato PR, Rivera F: Paternal involvement and children's behavior problems. J Marriage Fam 1999, 61(2):375-384.

11. Argys LM, Peters HE, Brooks-Gunn J, Smith JR: The impact of child support on cognitive outcomes of young children. Demography 1998, 35(2):159-173.

12. Kroll ME, Carson C, Redshaw M, Quigley MA: Early father involvement and subsequent child behaviour at ages 3,5 and 7 years: prospective analysis of the UK Millennium Cohort Study. PLoS One 2016, 11(9):e0162339.

13. Sarkadi A, Kristiansson R, Oberklaid F, Bremberg S: Fathers' involvement and children's developmental outcomes: a systematic review of longitudinal studies. Acta Paediatr 2008, 97(2):153-158.

14. Fagan J, Day R, Lamb ME, Cabrera NJ: Should researchers conceptualize differently the dimensions of parenting for fathers and mothers? J Fam Theory Rev 2014, 6(4):390-405.

15. Flouri E, Midouhas E, Narayanan MK: The relationship between father involvement and child problem behaviour in intact families: a 7-year cross-lagged study. J Abnorm Child Psychol 2016, 44(5):1011-1021.

16. McMunn A, Martin P, Kelly Y, Sacker A: Fathers' involvement: correlates and consequences for child socioemotional behavior in the United Kingdom. Journal of Family Issues 2017, 38(8):1109-1131.

17. Lengua LJ: Growth in temperament and parenting as predictors of adjustment during children's transition to adolescence. Dev Psycho/2006, 42(5):819832.

18. Chang LR, Chiu YN, Wu YY, Gau SSF: Father's parenting and father-child relationship among children and adolescents with attention-deficit/hyperactivity disorder. Compr Psychiat 2013, 54(2):128-140.

19. Shwalb DW, Shwalb BJ: Fatherhood in Brazil, Bangladesh, Russia, Japan, and Australia. Online Readings in Psychology and Culture 2014, 6(3):4.

20. Nishioka H: Men's domestic role and the gender system: determinants of husband's household labor in Japan ("Daiikkai zenkokukateidoukouchousa" data riyouniyorujisshotekikenkyu". Jinko Mondai Kenkyu (Journal of Population Problems) 1998, 54(3):56-71.

21. Nakazawa J, Shwalb DW: Fathering in Japan: entering an era of involvement with children. In: Fathers in cultural context. edn. Edited by Shwalb DW, Shwalb BJ, Lamb ME. New York: Routledge; 2013: 42-66.

22. Sato R, Fujiwara T, Kino S, Kawachi I: The association between father involvement in caregiving and early childhood overweight or obesity. Pediatr Obes 2020:e12652. 
23. Yorifuji T, Kubo T, Yamakawa M, Kato T, Inoue S, Tokinobu A, Doi H: Breastfeeding and behavioral development: a nationwide longitudinal survey in Japan. J Pediatr 2014, 164(5):1019-1025.

24. Okuzono S, Fujiwara T, Kato T, Kawachi I: Spanking and subsequent behavioral problems in toddlers: a propensity score-matched, prospective study in Japan. Child Abuse Neglect 2017, 69:62-71.

25. Cabrera N, Fitzgerald HE, Bradley RH, Roggman L: Modeling the dynamics of paternal influences on children over the life course. App/ Dev Sci 2007, 11(4):185-189.

26. Brown GL, McBride BA, Bost KK, Shin NN: Parental involvement, child temperament, and parents' work hours: Differential relations for mothers and fathers. J Appl Dev Psychol 2011, 32(6):313-322.

27. Hines M: Sex-related variation in human behavior and the brain. Trends in Cognitive Sciences 2010, 14(10):448-456.

28. Baxter J, Smart D: Fathering in Australia among couple families with young children. Australian Department of Families, Housing, Community Services and Indigenous Affairs, Occasional Paper 2011(37).

29. Amato PR, Rivera F: Paternal involvement and children's behavior problems. J Marriage Fam 1999:375-384.

30. Lewis SN, West AF, Stein A, Malmberg LE, Bethell K, Barnes J, Sylva K, Leach P, Team FP: A comparison of father-infant interaction between primary and non-primary care giving fathers. Child Care Health and Development 2009, 35(2):199-207.

31. Talge NM, Neal C, Glover V, Early stress trapsnfaneocaamh: Antenatal maternal stress and long-term effects on child neurodevelopment: how and why? Journal of Child Psychology and Psychiatry 2007, 48(3-4):245-261.

32. Crnic KA, Gaze C, Hoffman C: Cumulative parenting stress across the preschool period: relations to maternal parenting and child behaviour at age 5 . Infant Child Dev 2005, 14(2):117-132.

33. Paquette D: Theorizing the father-child relationship: mechanisms and developmental outcomes. Hum Dev 2004, 47(4):193-219.

34. Baker M, Milligan K: Boy-girl differences in parental time investments: evidence from three countries. J Hum Cap 2016, 10(4):399-441.

35. Harewood T, Vallotton CD, Brophy-Herb H: More than just the breadwinner: the effects of fathers' parenting stress on children's language and cognitive development. Infant Child Dev 2017, 26(2):19.

36. Emmott EH, Mace R: Why the son-bias in caregiving? testing sex-differences in the associations between paternal caregiving and child outcomes in England. Journal of Family Issues 2020, 30.

37. White Paper on Gender Equality 2019. In. Edited by Office GEBC. Tokyo: Cabinet Office; 2019.

38. JILPT Databook of International Labour Statistics 2018. In. Tokyo: The Japan Institute for Labour Policy and Training; 2018.

39. Fagan J, Lee Y: Perceptions and satisfaction with father onvolvement and adolescent mothers' postpartum depressive symptoms. Journal of Youth and Adolescence 2010, 39(9):1109-1121.

40. Kwok S, Cheng L, Chow BWY, Ling CCY: The spillover effect of parenting on marital satisfaction among chinese mothers. Journal of Child and Family Studies 2015, 24(3):772-783.

41. Musser AK, Ahmed AH, Foli KJ, Coddington JA: Paternal postpartum depression: what health care providers should know. J Pediatr Health Care 2013, 27(6):479-485. 